\title{
sciendo
}

Transport and Telecommunication, 2019, volume 20, no. 4, 318-324

Transport and Telecommunication Institute, Lomonosova 1, Riga, LV-1019, Latvia

DOI 10.2478/ttj-2019-0026

\section{MAKING WAREHOUSE LOGISTICS SMART BY EFFECTIVE PLACEMENT STRATEGY BASED ON GENETIC ALGORITHMS}

\author{
Aleksandrs Avdekins ${ }^{1}$ and Mihails Savrasovs ${ }^{2}$ \\ ${ }^{l} R \& D$ department manager, Trialto Latvia SIA, Riga, Latvia \\ ${ }^{2}$ Transport and Telecommunication Institute, Riga, Latvia \\ avdey@avdey.lv
}

\begin{abstract}
Supply chain executives are faced with the challenge of reducing labor costs. Travel time or picking efficiency can easily account for $50 \%$ or more of order picking time. If we omit human factor and the technical equipment of the warehouses, picking efficiency is mostly affected by two factors: correct combining orders into a single travel instance and picking orders in batch is the first factor; the second one is a goods placement - the more effective the goods are located, the shorter will be the picking distance for each order or batch of orders. It means that individual orders will be picked faster. Usually to determine the correct location for the goods 3PL's are using ABC analysis that includes indicators like count of orders, goods turnover, picking rate, weight etc.

There are also more complicated indicators like goods adjacency. Such indicators are harder to take into account using $\mathrm{ABC}$ analysis, as it requires sophisticated analysis of customer orders.

In recent publication goods placing by results of $\mathrm{ABC}$ analysis was compared to the genetic algorithm approach. It was showed that genetic algorithm much more effective for goods placing.

The goal of this paper is to improve developed genetic algorithm and include in calculations factors of the labor costs and warehouse topology. These factors will make algorithm usable in real warehouses and WMS (warehouse management system) information systems.
\end{abstract}

Keywords: Logistics, Genetic Algorithm, Optimization, Labour costs, Warehouse layout, Location assignment, Picking

\section{Warehouse layout optimization}

The basic warehouse operations are receiving, put away, storing, picking and shipping. Each of these operations consists of many smaller tasks and operations. Now, when demands of having efficient and lean warehouse are really high it is very important to have each of these operations on very effective level. Picking is the most costly process out the all other processes. The picking takes about $55 \%$ of the warehouse operating costs (Bartholdi and Hackman, 2011) (according to Koster et al. (2007) it is 50\% $70 \%$ ) and consists of: Traveling (55\%), Searching (15\%), Extracting (10\%), and Paperwork (20\%). This is the reason why companies trying to decrease picking costs. There are a lot of ways to achieve it. The most trivial way is to reduce labour costs and optimize warehouse shifts. This approach works only while there are room for optimisation sometimes it is not possible to reduce labour costs due to the fact other competitors pays more and workforce will move to the competitors. Another way to increase picking efficiency is to force pickers pick more goods and complete more picklists.

The time to pick an order or picklist can be divided into three components: time for travelling between items, time for picking the items, and time for remaining activities. The fact that about $50 \%$ of total order-picking time is spent on travelling (Tompkins et al., 1996) has the potential for improving the order-picking efficiency by reducing travel distances.

The correct goods placing on the warehouse allow to significantly decrease travelling distance of the picker. On the Figure 1 indicated possible picker route for the picklist consisting of 7 picks.

The picking process is chaotic and sometime picker forced to go back or to the opposite end of the warehouse to pick the item. Such picking behaviour could happen by the several reasons. Picking sequence or pickers route not optimized. Even WMS system will be forced to optimize sequence, picking time still can be optimized by shortening total picking distance. This can be achieved by changing some SKU's (stock keeping unit) placement closer to the docking area. 


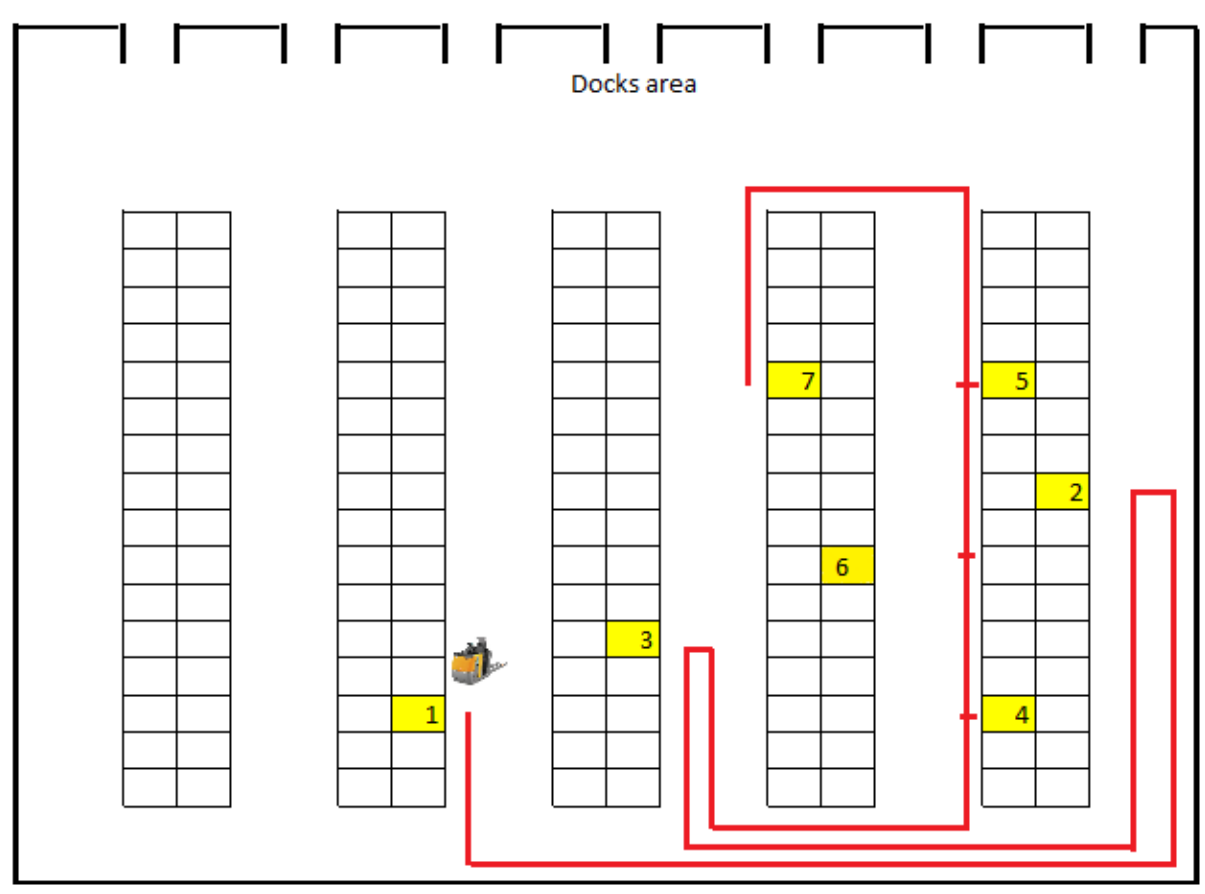

Figure 1. Picker route with bad goods placement

On the Figure 2 indicated route of the same picklist but after items were placed according its groups using $\mathrm{ABC}$ analysis. It is clear that second route much shorter and faster.

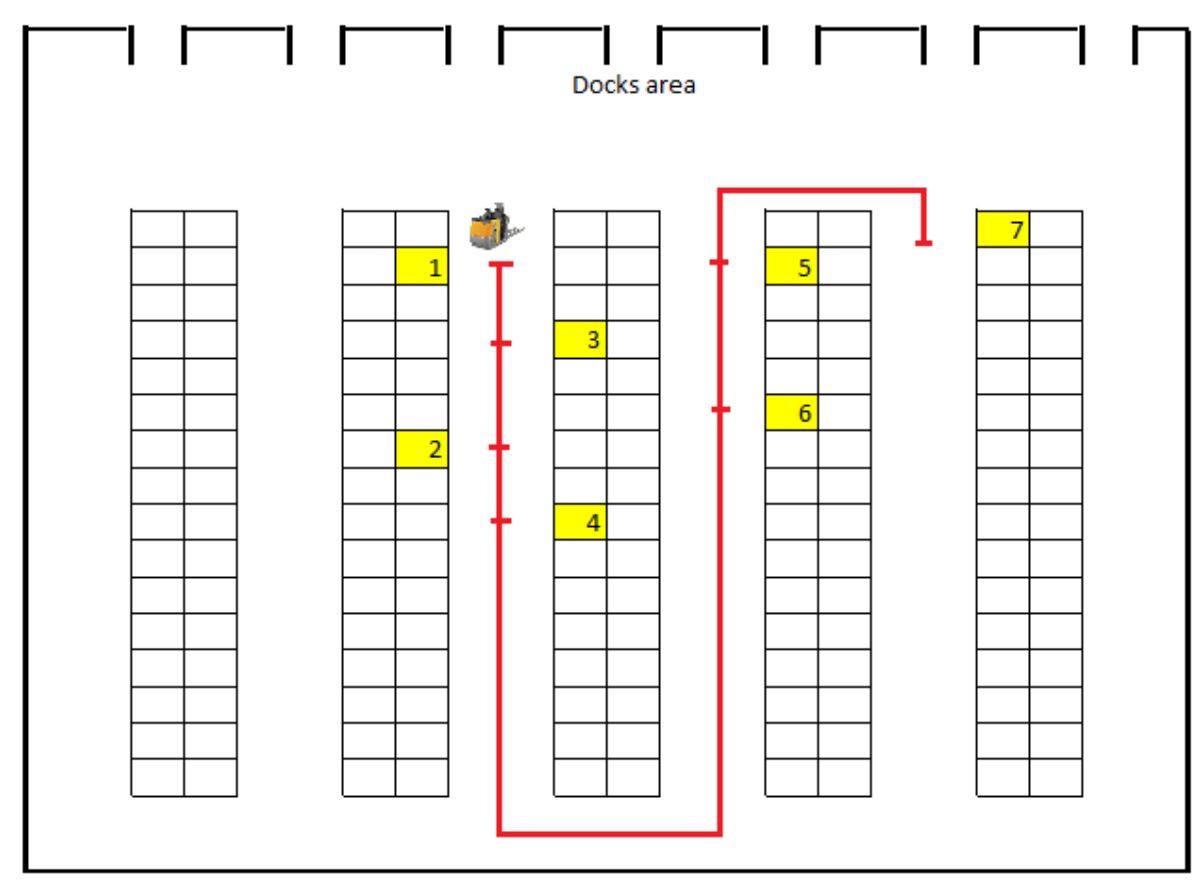

Figure 2. Picker route with better goods placement

Now it is clear, to pick faster we need to place goods in the way to minimize pickers travelling distance by optimizing picking sequence and reallocating SKU's. To find the optimal location for the SKU proposed to use genetic algorithm.

Genetic algorithm was used to solve warehouse location assignment problem for automated warehouses (Wang et al., 2017; Bei, 2016). In this paper problem is addressed for SME (small and 
medium enterprise) business segment where automation level not so high. Tonelli et al. (2002) in their publication concluded the necessity of using GA approach for goods placement. Developed GA showed good performance, efficiently determining an effective warehouse layout.

\section{Travelling salesman problem}

Evolutionary algorithms are the ones that follow the Darwin concept of "Survival of the fittest" mainly used for optimization problems for more than four decades (Holland, 1975).

Genetic algorithm (GA) is a search and optimization technique that mimics natural evolution. GA has already a relatively old history since the first work of John Holland on the adaptive systems goes back to 1962 ((Holland, 1962).

Genetic algorithms are by nature adaptive optimization algorithms that mimic the process of natural selection and genetics (Goldberg, 1989).

In GA terminology, a solution $\mathrm{x}$ is called an individual or a chromosome. Chromosomes are made of discrete units called genes. The main operations of a GA are: selection, crossover and mutation.

The Travelling Salesman Problem or the TSP is a representative of a large class of problems known as combinatorial optimization problems (Greco, 2008). The problem with warehouse picking is very similar to TSP, as every picker picking goods for each order is like a salesman that needs to find the best route.

The most popular practical application of TSP are: regular distribution of goods or resources, finding of the shortest of customer servicing route, planning bus lines etc., but also in the areas that have nothing to do with travel routes (Brezina and Cickova, 2011).

\subsection{Chromosome Representation}

Each chromosome coded to represent a solution for the defined problem - warehouse layout. Each gene is a unique item, that position in the chromosome represents the picking order of the items in the warehouse. If there are $\mathrm{n}$ items in the warehouse, so the chromosomes will look like shown in Table 1.

Table 1. Item representation in chromosomes

\begin{tabular}{lcccc}
\hline Chromosome 1: & & & \\
Item1 & Item2 & $\ldots$ & Item(n-1) & Item(n) \\
\hline Chromosome 2: & Item105 & $\ldots$ & Item(n-1) & Item(n) \\
Item22 & & & \\
\hline
\end{tabular}

Each chromosome gene order is the warehouse picking sequence, a route that will be used to pick each order.

\subsection{Initial Population, fitness and selection}

The initial population consist of 100 randomly generated individuals (chromosomes). Each chromosome is randomly filled with $\mathrm{n}$ items and each gene is unique (permutation encoding), as there is no need to have duplicate picking locations for items.

The fitness of each chromosome is calculated as the sum of maximal picking distance for each order. Each order picking distance is calculated from 0 to the furthest item picking location, where distance is item's position in the chromosome. Distance units of measure is an integer value that for the first item is 1, for second is 2 and etc., increasing by 1 from one SKU to another. Every chromosome fitness function calculated with Equation 1.

$\mathrm{f}\left(C_{x}\right)=\sum O_{k} d_{\max }(I)$

where $\mathrm{O}$ is order from the set $\{1,2 \ldots \mathrm{k}\}$, and the $\mathrm{d}_{\max }(\mathrm{I})$ is the distance to furthest picking position. 
Selection operation is performed based on the fitness function of each chromosome. This operation is required so that strongest individuals would participate in the crossover to get the better children (next generation). As it is required to minimize $\mathrm{f}$, it was selected only $30 \%$ of the population that have $\mathrm{f}->\mathrm{min}$ for crossover. Besides of granting a crossover change only for best current population chromosomes, the probability of participating in crossover is higher for the best of the best.

\subsection{Crossover and Mutation}

To create the next generation of the population an Order Crossover $(\mathrm{OX})$ is chosen as it is used for chromosomes with permutation encoding (Mitchell, 1998). The process starts by choosing two crossover points. It copies the subsequence of permutation elements between the crossover points from the cut string directly to the offspring, placing them in the same absolute position (Sivanandam and Deepa, 2007). To fill the rest of chromosome a sliding motion is applied.

Crossover principal on 10 genes example is presented in Figure 3.

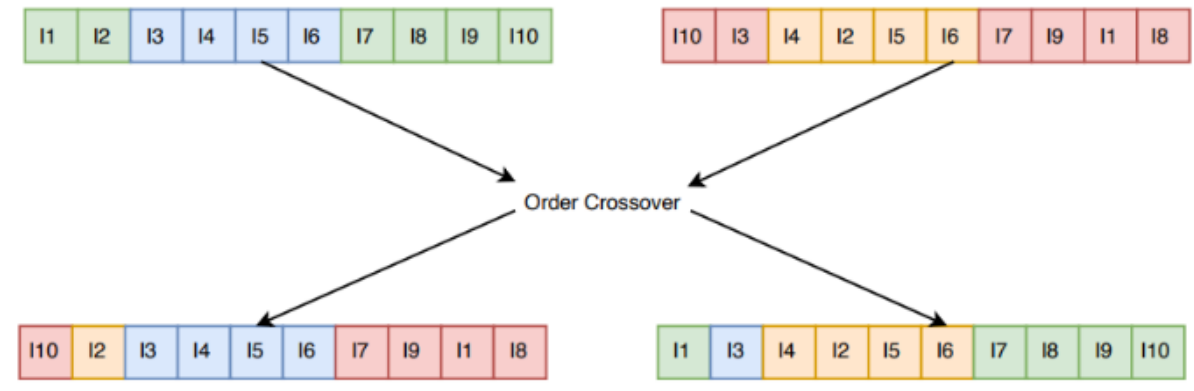

Figure 3. Crossover example

Selection and crossover are evolutionary operations that improve next population step by step, but there is a third operation that can drastically change chromosome's fitness. It is called mutation. It should be used to mutate $5 \%$ of each new population as this operation can change chromosome fitness to good or to worse; nevertheless, it adds a new solution to population, to help the algorithm not to stuck in local optimum.

\section{GA simulation results}

As input parameters of GA were used 974 unique items from 8802 orders with 117447 order lines. The initial population were 100 chromosomes. After 100 iterations, in case of average fitness function minimization result was less than $1 \%$ for last 10 iterations, the algorithm stops. Table 2 shows a summary of input parameters for GA algorithm.

Table 2. GA input parameters

\begin{tabular}{ll}
\hline Input parameter & Input value \\
\hline Population & 100 \\
Chromosome length & 974 genes \\
Fitness function & 8802 orders \\
Stop rule & Fitness improvement less than $1 \%$ \\
Simulation max iterations & 1000 \\
Simulations count & 10 \\
\hline
\end{tabular}

Each of the simulation finished on 1000 iteration and the best chromosome fitness score indicated in Table 3. 
Table 3. GA simulation running results

\begin{tabular}{llll}
\hline Simulation Nr. & Number of iterations & Best fitness function result & Run Time \\
\hline 1 & 986 & 483764 & $03: 30: 00$ \\
2 & 962 & 416557 & $03: 30: 00$ \\
3 & 964 & 508526 & $03: 55: 00$ \\
4 & 957 & 438915 & $02: 15: 00$ \\
5 & 998 & 476314 & $02: 13: 00$ \\
6 & 956 & 523760 & $02: 16: 00$ \\
7 & 827 & 525924 & $06: 05: 00$ \\
8 & 973 & 541549 & $06: 10: 00$ \\
9 & 981 & 512207 & $06: 05: 00$ \\
10 & 984 & 504699 & $06: 15: 00$ \\
\hline
\end{tabular}

The best fitness score GA achieved in the second simulation with 962 iterations (generation). Results for the best simulation are shown in Figure 4, where $\mathrm{X}$ is iteration number, $\mathrm{Y}$ is fitness score and $\mathrm{Z}$ is chromosome index.

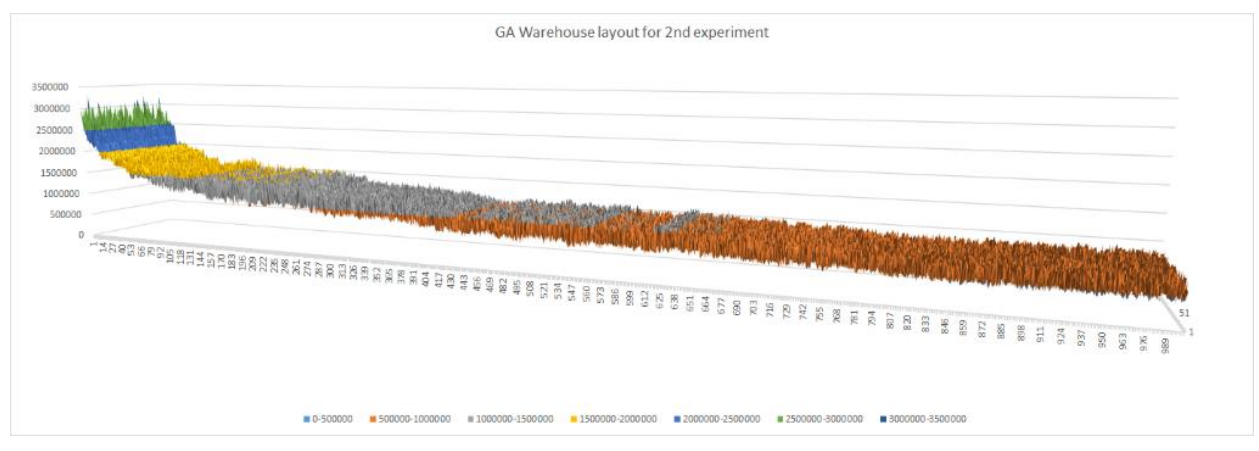

Figure 4. Best GA simulation progress

The graph on figure 2 illustrates that the solution was constantly improved. GA simulation fitness improvement represents an exponential decay function like experiences curve (sometimes called Henderson's Law). All 10 runs have similar fitness improvement curve, but with different learning rate.

Figure 5 demonstrates the $2 \mathrm{D}$ algorithm progression, where $\mathrm{X}$ is iteration number and $\mathrm{Y}$ is fitness score.

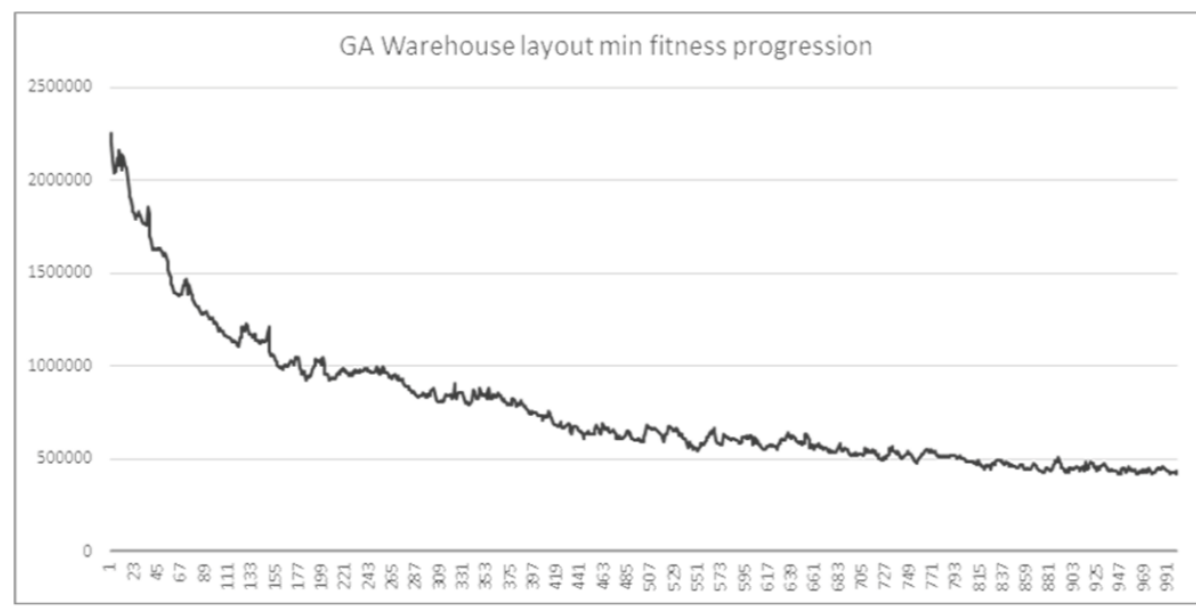

Figure 5. GA evolution 
The best fitness function score 416557 calculated for chromosome at 962 iterations. In the real world, that means the optimal items locations for the given order structure were found.

\section{GA results usage}

As a result of GA new suggested picking sequence were made. To apply these results in the existing warehouse it is needed to reallocate SKU from top of the sequence closer to the docks in order to pick faster. New warehouse layout with a new picking route for the same order looks like shown on the Figure 6. In green colour indicated warehouse part which is closer to the docks area where goods to be placed for packing. To make way of the picker even more optimal special aisle was introduced in the middle of each row. Picker got possibility to turn to the next aisle without driving to the end of the current row. Picking places was reallocated on the warehouse in green area according GA suggestion starting from the top left corner.

To avoid production stop and heavy goods reallocation costs suggested to apply Pareto principle to the GA results. First reallocate $20 \%$ of the goods taken from the top of the chromosome. Such approach allows to get picking time improvement without heavy investments into reallocation. In some time after reallocation when new picking data available GA can be applied again to find better goods locations taking in consideration changes done before. Several iterations with running GA and reallocation goods using Pareto principle can be performed in a period of time.



Figure 6. Picking sequence after GA results apply

\section{Conclusion}

Developed solution was tested on the data from the real warehouse and compared to the real picking process. After relocation to the new locations picking time decreased up to $60 \%$ comparing to the picking time before reallocation. To achieve such results helped not only goods reallocation but also new aisle introduction in the middle of the row to let picker move faster between the aisles.

To avoid heave investment in the labour costs reallocation was done in several steps using Pareto principle which also helped to get immediate results.

This research and experiment shows that GA approach can be applied on the warehouses with some adaptations of the physical layout - in our case aisle in the middle of the row was introduced. 


\section{References}

1. Avdeikins, A., Simakovs, A., Savrasovs, M. (2018) Genetic algorithm in comparison to ABC analysis for warehouse picking area layout calculation. In: RESEARCH and TECHNOLOGY - STEP into the FUTURE, 13(2), 67-73. Available:

http://www.tsi.lv/sites/default/files/editor/science/Conferences/RatSif_2018/rt_sf_2018_vol13_n2.pdf

2. Bartholdi, J. J., Hackman, S. T. (2011) Warehouse \& Distribution Science. Release: 0.95. Georgia Institute of Technology, School of Industrial and Systems Engineering, The Supply Chain and Logistics Institute. August 22 2011. Available: https://www.coursehero.com/file/16835153/wh-sci095/

3. Koster, R. D., Le-Duc, T. and Roodbergen, K. J. (2007) Design and control of warehouse order picking: A literature review. European Journal of Operational Research, 182 (2), 481-501. Available: http://www.sciencedirect.com/science/article/ pii/S0377221706006473

4. Holland, J. Adaptation in natural and artificial systems. Ann Arbor: University of Michigan Press (1975).

5. Holland, J. (1962) Outline for a logical theory of adaptive systems. J. Assoc. Comput. Mach., 9(3), 297-314.

6. Goldberg, D.E. (1989) Genetic algorithms in search, optimisation, and machine learning. Addison Wesley Longman.

7. Greco, F. (2008) Travelling Salesman Problem. In-The. Available: http://www.exatas.ufpr.br/portal/docs_degraf/paulo/TravellingSalesmanProblem.pdf

8. Brezina, I., Cickova, Z. (2011) Solving the Travelling Salesman Problem using the Ant colony Optimization. Management Information Systems, 6(4), 010-014. Available: http://www.ef.uns.ac.rs/mis/archive-pdf/2011\%20-\%20No4/MIS2011_4_2.pdf

9. Mitchell, M., Davis, L.D. (1998) Handbook of Genetic Algorithms. Artificial Intelligence, 100, 325-330. Available: https://reader.elsevier.com/reader/sd/pii/S0004370298000162?token= EF6DDF6CDBAC210CAD1AFC76FDC959FB431860C0BCF6AED340B2E21130C793EF6A9B0D BB1C67BFA0069BD8296B4805E3

10. Sivanandam, S.N., Deepa, S. N. (2007) Introduction to Genetic Algorithms. Springer.

11. Tompkins, J.A, White, J.A, Bozer, Y.A, Frazelle E.H. (1996) Facilities Planning. $2^{\text {nd }}$ ed. John Wiley \& Sons, NY.

12. Wang, W, Gao, J., Gao, T., Zhao, H. (2017) Optimization of Automated Warehouse Location Based on Genetic Algorithm. In: Advances in Intelligent Systems Research, 134, 309-313. Available: https://www.atlantis-press.com/proceedings/caai-17/25881178

Bei, R. (2016) A Optimization Study on Irregular Positions in Automatic Warehousing Systems. In: International Journal of Simulation - Systems, Science \& Techno, 17(48), 1-2. Available: https://pdfs.semanticscholar.org/5bbd/88fb8bb262e48d001454c72acfa9d897fb5a.pdf

13. Tonelli, F., Schenone, M., Nan, P., Zunino, I. (2003) Warehouse Layout Design: Minimizing Travel Time with a Genetic and Simulative Approach - Methodology and Case Study. In: Proceedings 14th European Simulation Symposium. Available: https://www.researchgate.net/publication/2897817_Warehouse_layout_design_minimizing_travel_ti me_with_a_genetic_and_simulative_approach-methodology_and_case_study 